\title{
Sleep EEG maps the functional neuroanatomy of executive processes in adolescents born very preterm
}

Flavia M. Wehrle ${ }^{\mathrm{a}, \mathrm{b}}$, Beatrice Latal ${ }^{\mathrm{b}, \mathrm{c}}$, Ruth L. O'Gorman ${ }^{\mathrm{c}, \mathrm{d}, \mathrm{e}}$, Cornelia F. Hagmann ${ }^{\mathrm{a}}$, Reto Huber $^{\mathrm{b}, \mathrm{c}, \mathrm{f}}$

${ }^{\mathrm{a}}$ Department of Neonatology, University Hospital Zurich, Frauenklinikstrasse 10, 8091 Zürich, Switzerland; ${ }^{\mathrm{b}}$ Child Development Center, University Children's Hospital Zurich, Steinwiesstrasse 75, 8032 Zürich, Switzerland; 'Children's Research Center, University Children's Hospital Zurich, Steinwiesstrasse 75, 8032 Zürich, Switzerland; 'Center for MR Research, University Children's Hospital Zurich, Steinwiesstrasse 75, 8032 Zürich, Switzerland; e Zurich Center for Integrative Human Physiology, University of Zurich, Winterthurerstrasse 190, 8057 Zürich, Switzerland; f Department of Child and Adolescent Psychiatry and Psychotherapy, Psychiatric Hospital, University of Zurich, Neumünsterallee 9, 8032 Zürich, Switzerland

Address of corresponding author: Reto Huber, Child Development Center, University Children's Hospital Zurich, Steinwiesstrasse 75, 8032 Zurich, Switzerland, Tel.: +41 (0)44 2668160 , Email: reto.huber@kispi.uzh.ch

\section{Abbreviations:}

ANCOVA - Analysis of Covariance; CANTAB - Cambridge Neuropsychological Test Automated Battery; FDR - false discovery rate; NREM sleep - non-rapid eye movement sleep; RWT - Regensburger Wortflüssigkeitstest; SES - Socio-economic status; SnPM - Statistical nonparametric mapping; SWA - Slow wave activity 


\begin{abstract}
Executive function deficits are among the most frequent sequela of very preterm birth but the underlying neuronal mechanisms are not fully understood. We used high-density EEG recordings during sleep to assess alterations in the functional neuroanatomy of executive processes in adolescents born very preterm. The topographical distribution of sleep slow wave activity (SWA; 1-4.5 Hz EEG power) has previously been used to map cognitive abilities and is known to reflect the intensity of the prior use of the respective neuronal networks. We assessed 38 adolescents born before 32 weeks of gestation (age at assessment: 12.9 (SD: 1.7), range: 10.616.7 years) and 43 term-born peers (13.1 (2.0), 10.0-16.9). Executive function abilities were quantified with a composite score derived from a comprehensive task battery. All-night highdensity EEG (128 electrodes) was recorded and SWA of the first hour of sleep was calculated. Abilities were significantly poorer in the very preterm compared to the term-group, particularly, if the tasks demands were high $(P<.01)$. The score was positively correlated with sleep SWA in a cluster of 15 electrodes over frontal and negatively in a cluster of 14 electrodes over central brain regions after controlling for age at assessment and correcting for multiple comparisons. Within the frontal cluster, sleep SWA was higher in very preterm compared to term-born participants when controlling for executive function performance and age at assessment $(P=.02)$. No difference in SWA between very preterm and term-born participants was found for the central cluster $(P=.29)$. Our results demonstrate a local increase of sleep SWA over brain regions associated with executive processes in adolescents born very preterm compared to similarly performing term-born peers. Thus, sleep SWA maps the higher effort needed for executive function tasks in adolescents born very preterm.
\end{abstract}

\title{
Keywords:
}

Sleep, slow wave activity, executive functions, prematurity, functional neuroanatomy 


\section{Introduction}

Very preterm birth (<32 weeks of gestation) is a significant risk factor for neurodevelopmental deficits in childhood and adolescence (Latal, 2009). Particularly, executive functions, a set of higher-order cognitive abilities needed for goal-directed behavior (e.g., inhibition, working memory, cognitive flexibility (Miyake et al., 2000; Anderson, 2002)), are frequently impaired in those born very preterm (Aarnoudse et al., 2012; Burnett et al., 2015). We have shown recently that even very preterm children and adolescents with normal intellectual and motor abilities may be affected when the demands placed on their abilities are high (Wehrle et al., 2016).

Impaired structural and functional neuroanatomy due to early disruptions of normal brain development have been suggested to underlie the neurodevelopmental deficits associated with preterm birth (Ment and Constable, 2007; Ment et al., 2009; Volpe, 2009). More specifically, impaired executive function performance was found to be related to smaller regional grey and white matter volumes (Nosarti et al., 2008; Taylor et al., 2011), reduced cortical thickness (Skranes et al., 2012) and impaired white matter microstructure (Skranes et al., 2009) in very preterm born children and adolescents. In parallel, using functional MRI, altered neural activation patterns in response to executive function tasks have been reported (Curtis et al., 2006; Nosarti et al., 2006; Griffiths et al., 2013; Mürner-Lavanchy et al., 2014). However, alterations in the functional neuroanatomy of executive processing was associated with both task-related (e.g., cognitive load of the task) and subject-related (e.g., individual cognitive capacity) factors and, thus, may limit the interpretation of the findings (Murphy and Garavan, 2004; Ment and Constable, 2007; Uddin et al., 2010). In contrast, assessing brain activity during sleep allows the investigation of the functional anatomy of neurocognitive networks independent of factors related to wakefulness (e.g., motivation or concentration), as during sleep, individuals are virtually disconnected from the environment (Tononi and Cirelli, 2006). Importantly, high-density electroencephalography (EEG) allows the investigation of topographical patterns of brain activity by combining the superior temporal resolution of EEG recordings with high spatial resolution (Lustenberger and Huber, 2012).

The topographical distribution of sleep EEG activity has been suggested to reflect individual traits of functional neuroanatomy as it is highly stable within but varies considerably between individuals (Finelli et al., 2001; De Gennaro et al., 2005; Geiger et al., 2012; 
Lustenberger et al., 2016). Sleep slow wave activity (SWA; EEG spectral power between 1 and 4.5 Hz during non-rapid eye movement (NREM) sleep) may be of particular interest when investigating functional neuroanatomy as it is a marker of neural synchrony and synaptic strength within cortical networks (Tononi and Cirelli, 2006). SWA has previously been used to map cognitive and motor skills as well as maturational aspects of functional neuroanatomy in typically-developing children and adolescents (Kurth et al., 2010; Kurth et al., 2012; Lustenberger et al., 2016). Further, SWA topography in pediatric populations (e.g., children with attention-deficit/hyperactivity disorder) was used to illustrate disease-related alterations in functional neuroanatomy compared to typical brain development (Ringli et al., 2013). It is known that sleep slow waves measured in the surface EEG are a reflection of the synchronized oscillatory activity of the thalamocortical system (Steriade et al., 1993; Crunelli et al., 2015), and this knowledge may help the interpretation of alterations in sleep SWA following very preterm birth.

In the current study, we aimed to investigate the association between SWA assessed by high-density EEG during sleep and executive functions. We hypothesized that SWA maps alterations in the functional neuroanatomy of executive processes in children and adolescents born very preterm compared to typically-developing term-born peers.

\section{Materials and methods}

\subsection{Participants and experimental design}

The eligibility criteria and the selection process for participation in the current study have been described in detail previously (Wehrle et al., 2016). In short, individuals between 10 and 16 years of age who were born very preterm ( $<32$ weeks of gestation), had not suffered from any major neonatal brain injuries and who showed normal intellectual and motor development at the age of five years were eligible for the current study. Of 175 eligible individuals, 41 consented to participate.. Perinatal and routine follow-up data were collected from the hospital's medical records. Those who agreed to participate did not differ from those who did not agree with regard to gestational age, birth weight, perinatal complications and intellectual and motor abilities assessed at the follow-up consultation (all $p>.05$ ). Forty-three typically-developing siblings and friends of very preterm participants and individuals from local schools were recruited as the 
control group. They were group matched to the very preterm participants in terms of sex and age at assessment. For all participants, socio-economic status (SES) was estimated using a six-point scale based on maternal education and paternal occupation (Largo et al., 1989).

One week prior to the assessment, participants were instructed to keep a regular sleepwake schedule according to their habitual bed time. Compliance was verified with self-reported sleep logs and wrist motor actigraphy (Actiwatch Plus, AW4, Cambridge Neurotechnology, Cambridge, England). Over the course of a full afternoon, cognitive abilities were assessed by an examiner who was aware of the birth status but unaware of the medical history of the participants. After dinner, the EEG recording was prepared and participants went to bed at their habitual bed time. Wake-up times were adjusted to the participants' individual routines (e.g., school attendance). All data were collected between January and December 2013 at the Child Development Center and the Sleep Laboratory of the University Children's Hospital Zurich. Assessments took place on weekdays, weekends and during holidays as was most convenient for participating families. The study was approved by the local ethical committee. Written informed consent was obtained from a parent as well as from participants older than 15 years. Younger participants provided oral consent. Participants were compensated with a gift certificate.

\subsection{Assessment of executive functions}

Detailed methods on the neurodevelopmental assessment in this cohort have been reported previously (Wehrle et al., 2016). In short, general intellectual abilities were estimated with an abbreviated version of the Wechsler Intelligence Scale for Children (WISC-IV, German version (Petermann and Petermann, 2006)) and motor abilities were assessed with the pegboard subtest of the Zurich Neuromotor Assessment (ZNA (Largo et al., 2007)). Executive functions were assessed with three subtests of the Cambridge Neuropsychological Test Automated Battery (CANTAB $(2004,2011)$ ) and the Regensburger Wortflüssigkeits-Test (RWT (Aschenbrenner et al., 2000)), a German-language verbal fluency test. The CANTAB consisted of the Stockings of Cambridge task to assess planning abilities, the Spatial Working Memory task to assess working memory and the Intra-/Extradimensional Shift task to assess cognitive flexibility. The RWT included phonetic and semantic fluency and switching subtests. The results of the subtests were averaged to reflect overall verbal fluency abilities. Each executive function task consisted of 
multiple trials with increasing levels of demand, for example one versus two minutes of word production in the RWT task (see Wehrle et al., 2016 for details).

An executive function composite score reflecting performance in highly demanding tasks was calculated, as deficits have been shown to become clinically relevant when task demands are high (Wehrle et al., 2016). For each of the four executive function tasks, the performance in low demand trials was subtracted from the performance in high demand trials to take into account individual baseline performance (i.e., performance when the demands placed on the abilities are low). Subsequently, results were z-transformed using the mean and standard deviation of the control group to obtain equal scaling of the different tasks. Z-scores were combined in a single composite score reflecting executive function abilities in highly demanding trials adjusted for baseline performance.

\subsection{All-night sleep EEG recording and preprocessing}

All-night EEG during sleep was recorded with a high-density EEG device with 128 channels (Electrical Geodesic Inc.; Sensor Net for long-term monitoring). The nets were adjusted to the vertex and gel electrolyte was used to fill the electrodes. Impedances were kept below 50 $\mathrm{k} \Omega$. EEG recordings were sampled at $500 \mathrm{~Hz}$ (filtered between 0.01 and $200 \mathrm{~Hz}$ ) and referenced to the vertex $(\mathrm{Cz})$. For further analyses, the data were band-pass filtered $(0.5-50 \mathrm{~Hz})$ and downsampled to $128 \mathrm{~Hz}$. Sleep stages were scored for 20 -second epochs according to standard criteria (Iber et al., 2007) by one person who was blinded to birth status. Artifacts were identified and the corresponding epochs were removed on a 20 -second basis by visual inspection and if power exceeded a threshold based on a mean power value in the $0.75-4.5$ or $20-30 \mathrm{~Hz}$ band (Lustenberger et al., 2014). Also, channels with overall bad quality were removed (on average one EEG channel per participant; range: 0 to 4). Data were re-referenced to the average of all good quality channels above the ears (in total 109 channels). Two participants born very preterm refused the EEG recording at the day of the assessment and for one participant born very preterm, data were lost due to technical difficulties. For two term-born participants, the majority of channels lost signal during the second half of the night and these participants were excluded for the analyses concerning these time points of the night. For one very preterm participant, the net was removed after 4.5 hours due to discomfort. Thus, complete datasets were available for further 
analyses in 38 very preterm and 43 term-born participants (37 and 41 for the analyses of later time points of the night, respectively).

\subsection{Spectral analysis, power analysis and statistical analyses}

Spectral analysis of consecutive 20-second epochs (fast Fourier transformation, Hanning window, average of five 4-second epochs, frequency resolution of $0.25 \mathrm{~Hz}$ ) was performed. SWA was calculated as the mean power in the frequency band between 1 and $4.5 \mathrm{~Hz}$ during the first hour of NREM sleep (stage N2 and N3). This interval was selected to account for inter-individual differences in sleep episode duration and because it constitutes the most consolidated part of sleep. Additionally, SWA during the last hour of NREM sleep was calculated to investigate the stability of the effects across the night. Absolute power values were normalized for each participant by dividing the power in each electrode by the average power of all good quality electrodes above the ears. Normalized power values were used for all analyses as they have been shown previously to reliably reflect local aspects of SWA (Kurth et al., 2010; Buchmann et al., 2011b).

To assess the association between SWA and executive function abilities, Pearson productmoment correlation coefficients between SWA in every electrode and the executive function composite score were calculated. To correct for multiple comparisons, statistical nonparametric mapping (SnPM) using a suprathreshold cluster analysis was applied (Nichols and Holmes, 2002; Huber et al., 2004). $R$-values within clusters were Fisher z-transformed and the mean correlation across the electrodes within the cluster was calculated. To assess whether the association between executive function abilities and EEG power within the identified clusters was specific to the SWA frequency range, the executive function composite score was correlated to the power in every $0.25 \mathrm{~Hz}$ frequency bin between 1 and $25 \mathrm{~Hz}$. To correct for multiple comparisons, a false discovery rate (FDR) correction was applied. Mean SWA of all electrodes within an identified cluster was calculated and compared between very preterm and term-born participants by means of univariate analyses of covariance (ANCOVA) with mean SWA within a cluster as the independent variable, birth status as a fixed factor and executive function performance and age at assessment as covariates. All analyses were performed with the software package MATLAB (MathWorks) and SPSS 22.0. The significance level was set at $p<.05$ (two-tailed). 


\section{Results}

3.1 Participant characteristics, executive function abilities and sleep architecture

The mean age at assessment was 12.9 (SD: 1.7; range: 10.6-16.7) and 13.1 (2.0; 10.0 16.9) years in the very preterm and term-born group, respectively $(p=.56)$. The sex distribution was not significantly different between the two groups (55.3 and $48.8 \%$ boys in the very preterm and term-born group, respectively, $p=.56)$. SES was significantly lower in the very preterm (2.5 $[1.0 ; 1-4])$ compared to the term-born group $(2.0[0.9 ; 1-4], p=.02$, with higher scores indicating lower SES). Very preterm participants were born at a mean gestational age of 29.5 (2.1; 25.132.0) weeks and with a mean birthweight of 1277 (348; 840-1990) grams. Major perinatal characteristics are summarized in Supplementary Table 1.

Estimated IQ scores were significantly lower in the very preterm compared to the termborn group (104.1 [7.3] and 109.5 [6.8], respectively, $p=.04$ after controlling for SES) with all participants scoring in the normal range (91-118 and 99-128, respectively). Fine motor abilities were not different between the groups (22.4 [4.6] and 22.2 [3.6] seconds to solve the pegboard in the two groups, respectively, $p=.58$ after controlling for SES). Executive function performance was good and similar in the two groups when the task demands were low but very preterm participants scored significantly poorer than their term-born peers when the demands increased (Figure 1a-d). Accordingly, the overall executive function composite score was significantly lower in very preterm compared to term-born participants $(F(1,77)=9.374, p=.003$ (adjusted for age at assessment and SES), Figure 1e). 
very preterm participants

$\square$ term-born participants

a

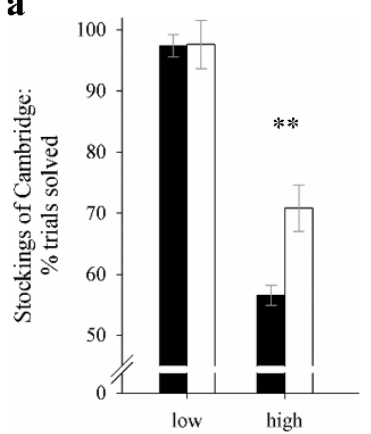

c

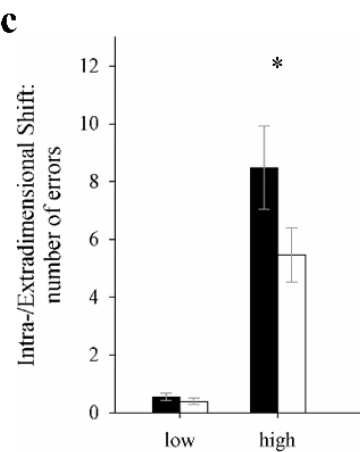

e

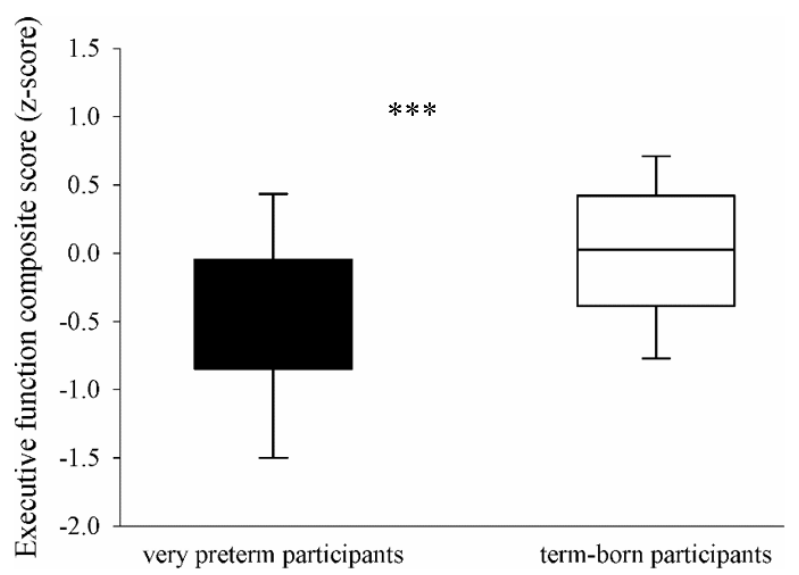

b

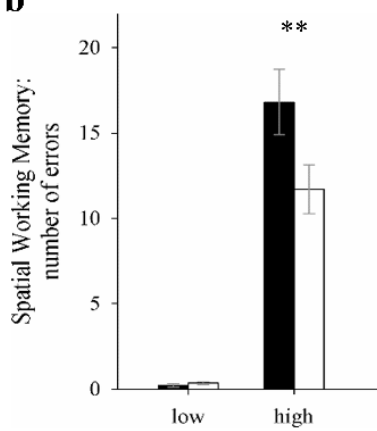

d

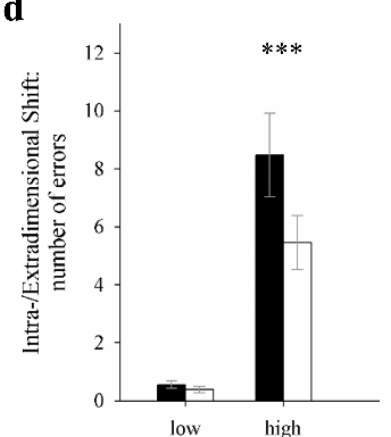


Sleep efficiency (i.e., time asleep as a percentage of time in bed) was high at $87.0 \%$ and $87.3 \%$ in the very preterm and the term-born participants, respectively $(p=.86)$. No significant difference between the groups was found in any of the assessed parameters of sleep architecture (Table 1). Global SWA during NREM sleep (EEG power averaged across all good-quality electrodes) was not significantly different between the groups, neither in the first nor in the last hour of the night $(F(1,78)=2.314, p=.13$ and $F(1,75)=3.144, p=.08$, adjusted for age at assessment). Moreover, when directly comparing the topographical distribution of SWA, no group differences were apparent (SnPM suprathreshold cluster analysis, data not shown).

Table 1. Visually scored parameters of sleep architecture.

\begin{tabular}{lccc} 
& Very preterm participants $(n=38)$ & Term-born participants $(n=43)$ & $p^{\mathrm{e}}$ \\
\hline Sleep efficiency $^{\mathrm{a}}$ & $87.0(9.1 ; 57.7-96.4)$ & $87.3(8.8 ; 60.3-97.6)$ & .86 \\
Total time in bed (in minutes) & $547.1(68.3 ; 271.7-649.0)$ & $544.3(41.0 ; 478.3-636.7)$ & .82 \\
Total sleep time (in minutes) $_{\text {Sleep latency (in minutes) }}^{\mathrm{b}}$ & $477.5(78.8 ; 156.7-586.7)$ & $475.5(60.6 ; 299.3-608.7)$ & .90 \\
REM sleep latency (in minutes) $^{\mathrm{c}}$ & $27.7(16.7 ; 6.0-78.3)$ & $24.7(16.3 ; 2.3-82.7)$ & .46 \\
NREM (in \% ${ }^{\mathrm{d}}$ ) & $146.8(59.4 ; 60.7-301.0)$ & $146.3(67.7 ; 58.0-301)$ & .97 \\
$\quad$ N1 & $76.1(3.3 ; 69.3-82.6)$ & $75.8(5.6 ; 63.8-88.7)$ & .75 \\
N2 & $11.2(4.7 ; 4.3-24.9)$ & $10.5(3.7 ; 3.8-20.8)$ & .41 \\
N3 & $50.1(5.9 ; 31.3-59.7)$ & $50.9(5.9 ; 43.1-64.5)$ & .56 \\
REM (in \% ${ }^{\mathrm{d}}$ ) & $14.8(4.1 ; 9.5-29.6)$ & $14.4(4.7 ; 3.4-29.4)$ & .74 \\
\hline
\end{tabular}

${ }^{a}$ Time asleep as percentage of total time in bed. ${ }^{b}$ First appearance of NREM sleep (N2). ${ }^{c}$ First appearance of REM sleep. ${ }^{\mathrm{d}} \%$ of total sleep time. ${ }^{\mathrm{e}}$ independent samples $t$-test. Mean (standard deviation; range).

\subsection{Regional association patterns of SWA and executive function abilities}

Based on our previous knowledge about functional deficits under high demand, we expected specific, task related differences in SWA. Thus, in a first step, we identified regions where SWA was associated with executive function abilities by pooling all subjects and performing electrode-wise Pearson correlations between SWA during the first hour of NREM sleep and the executive function composite score. Figure 2 shows the two clusters in which 
significant correlations between SWA and executive functions were found for the pooled group (SnPM suprathreshold cluster analysis): SWA in a cluster of 15 electrodes over frontal brain regions was positively associated with the executive function composite score. The mean correlation across the electrodes within this cluster was $r=.33$ (SD: .07; range: .23 to .47 ). In a central cluster of 14 electrodes, SWA was correlated negatively with the executive function composite score. The mean correlation across the electrodes within this cluster was $r=-.28$ (SD) .04 ; range: -.35 to -.24$)$.

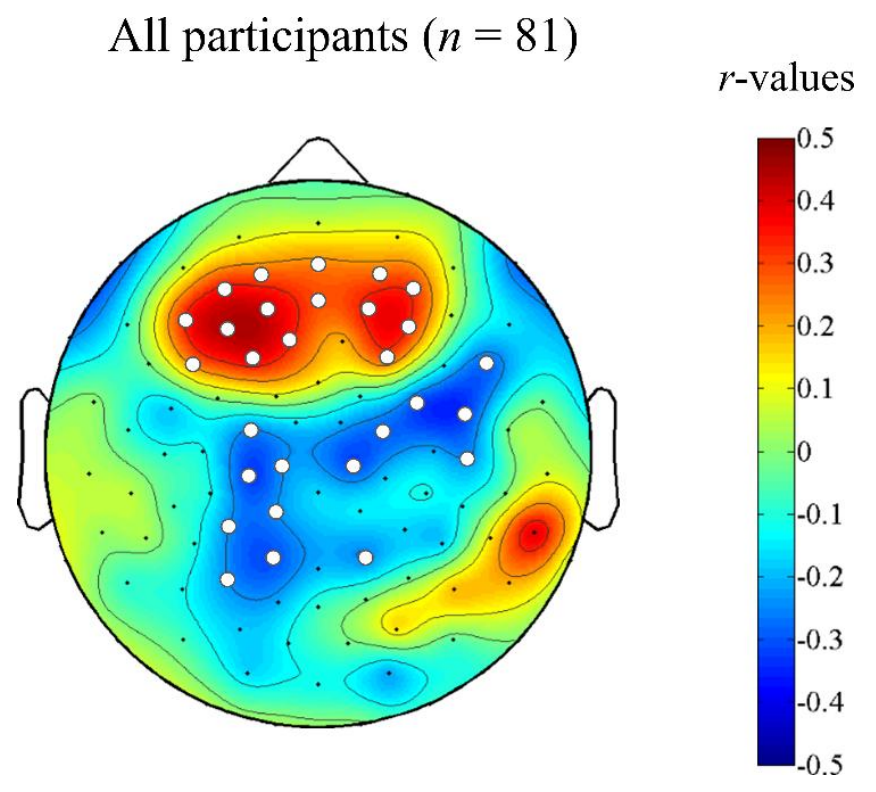

Figure 2. Association between sleep slow wave activity (SWA; EEG power between 1 and 4.5 $\mathrm{Hz}$, based on the first hour of NREM sleep stages N2 and N3) and executive function abilities across all participants. Results of the electrode-wise Pearson correlations between SWA and the executive function composite score plotted on the planar projection of the hemispheric scalp model. White dots indicate clusters of electrodes showing significant correlations (defined by statistical nonparametric mapping (SnPM; suprathreshold cluster analysis to control for multiple comparison (Nichols and Holmes, 2002)).

To assess the stability of the topographical pattern across the night, we correlated SWA of the last hour of NREM sleep at each electrode with the executive function composite score: This analysis revealed a positive trend-level correlation in a cluster of 9 frontal electrodes showing a significant correlation ( $p \leq .10$, SnPM suprathreshold cluster analysis) which overlapped with the 
cluster apparent during the first hour of NREM sleep (mean $r=.27$; SD: .04; range: .24 to .34). No significant cluster was identified over central brain regions.

The association between EEG power in the frontal and central cluster and the executive function composite score was specific to the SWA frequency range. In the frontal cluster, significant correlations were found exclusively in 12 frequency bins between 1 and $4 \mathrm{~Hz}$ after FDR-correction. In the central cluster, EEG power was associated with executive function performance exclusively in 8 frequency bins between 1 and $3.5 \mathrm{~Hz}$ after FDR-correction.

We also examined whether the regional association patterns between SWA and executive function abilities were different between the two groups. The positive associations between SWA and executive function abilities over frontal brain regions was apparent in both groups but tended to be more pronounced in the very preterm group. The topographical distribution of negative associations between SWA and executive function abilities over central brain regions was more variable between the groups (Figure 3).

Very preterm group $(n=38)$

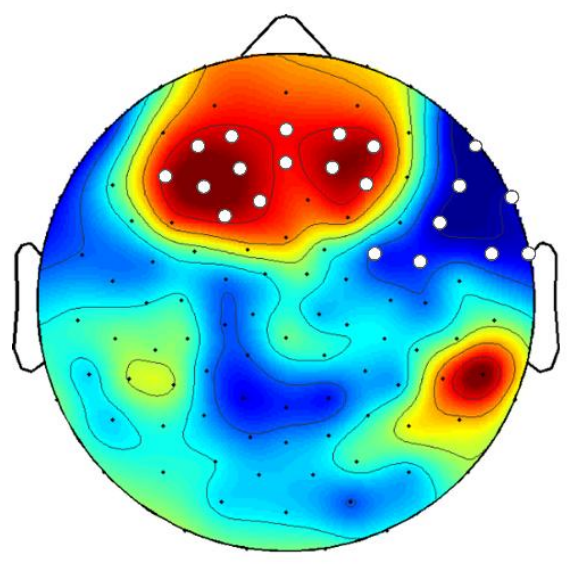

Term-born group $(n=43)$

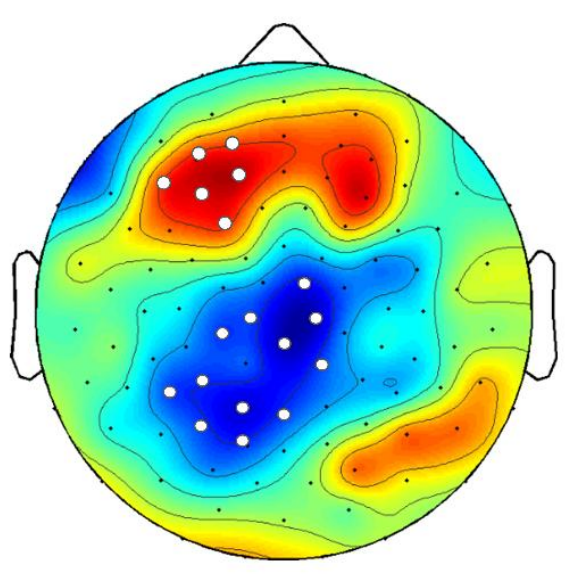

$r$-values

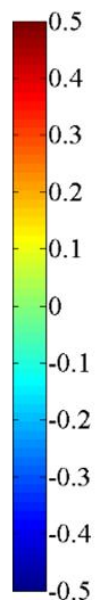

Figure 3. Association between SWA and executive function abilities separately for the very preterm and the term-born group. Very preterm group: frontal cluster of 13 and temporo-central of 8 electrodes showing significant correlations (mean $r=.46 ; S D: .07$; range: .36 to .61 and mean $r=-.48$; SD: .11; range: -.70 to -.34 , respectively). Term-born group: frontal cluster of 6 and central cluster of 12 electrodes showing significant correlations (mean $r=.38 ; S D$ : .05; 
range: .32 to $.46 ; p \leq .10, \mathrm{SnPM}$ suprathreshold cluster analysis and mean $r=-.38 ; S D: .06$; range: -.49 to -.32 , respectively).

3.3 SWA difference between very preterm and term-born participants in clusters of interest

Next, we assessed whether SWA in the frontal and central clusters of interest was different between the groups when holding executive function performance constant, i.e., controlling for individual performance levels. Mean SWA of the first hour of NREM sleep in the frontal cluster was significantly higher in the very preterm group compared to the term-born group when controlling for executive function performance and age at assessment $(F(1,77)=$ $5.324, p=.02$, Figure 4). No significant difference between the groups was found in the central cluster $(F(1,77)=1.123, p=.29)$. The results persisted when including SES as an additional covariate ( $p=.05$ for the frontal and $p=.44$ for the central cluster, respectively).

To assess the stability of this group difference across the night, the analyses were repeated with mean SWA of the last hour of NREM sleep as the dependent variable. SWA was not significantly different between the groups at the end of the night after adjusting for executive function performance and age at assessment, neither in the frontal nor in the central cluster $(F(1$, $74)=.094, p=.76$ and $F(1,74)=.002, p=.97$, respectively).

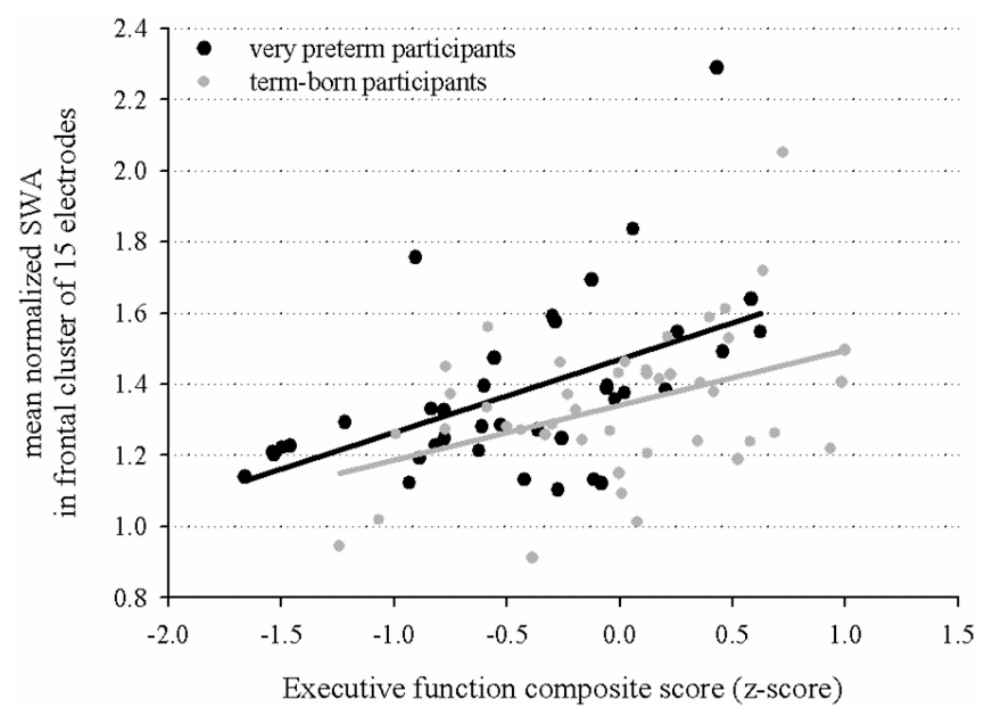


Figure 4. Association between executive function performance and mean sleep slow wave activity (SWA; normalized: absolute power in each electrode divided by average power of all good quality electrodes above the ears) in a cluster of 15 electrodes over frontal brain regions in very preterm $(n=38)$ and term-born $(n=43)$ participants. Significantly higher slow wave activity in the very preterm compared to the term-born group $(F(1,77)=5.324, p=.02$, adjusted for executive function performance and age at assessment with results persisting when including socio-economic status as an additional covariate $(p=.05))$.

\section{Discussion}

In this study, sleep SWA assessed with high-density EEG was used to map the functional neuroanatomy of executive processes in children and adolescents born very preterm in comparison to term-born peers. Positive associations between sleep SWA and executive function abilities were found in a widespread network of frontal brain regions. Within this area, individuals born very preterm exhibited more SWA compared to similarly performing term-born peers. Over central brain regions, SWA was negatively associated with executive function abilities while no difference between the groups was apparent in these regions.

The topographical distribution of the EEG power during deep sleep has been suggested to reflect individual traits of functional neuroanatomy as it is highly stable within individuals across multiple nights while it varies considerably between individuals (Finelli et al., 2001; De Gennaro et al., 2005; Geiger et al., 2012; Lustenberger et al., 2016). Twin studies have estimated its heritability to lie above $90 \%$, thus, making it to one of the most heritable traits of humans (De Gennaro et al., 2008). Particularly, sleep SWA topography is a useful tool to map neurofunctional networks of cognitive and motor abilities in typically-developing children and adolescents (Kurth et al., 2012; Pugin et al., 2014; Wilhelm et al., 2014). The results of the current study expand and advance these findings by demonstrating that SWA topography reliably maps the functional neuroanatomy of executive processes following very preterm birth.

The stable and frequency-specific positive association across the night between sleep SWA and executive function abilities over frontal brain regions is in line with a previous study which reported cognitive control skills to be positively related to sleep SWA over frontal brain regions in typically-developing children (Kurth et al., 2012). Also, structural and functional 
neuroimaging studies in both typically-developing and very preterm children and adolescents found frontal brain areas to be critically involved in executive function processing (Anderson, 2002; Diamond, 2002; Nosarti et al., 2008; Mürner-Lavanchy et al., 2014; Ullman et al., 2014; Østgård et al., 2016).

Identifying potential alterations in the functional neuroanatomy of executive processes following very preterm birth was of particular interest in the current study. The association between higher SWA over frontal brain regions and better executive function abilities could be detected both in the very preterm and in the term-born group, thus, suggesting overall similar functional neuroanatomy underlying executive processes in both groups. This is in line with reports from functional MRI studies which found activation in similar anatomical regions in very preterm and term-born individuals while performing executive function tasks (Griffiths et al., 2013; Daamen et al., 2014). Interestingly, the cluster of significant positive associations was more widespread and pronounced in the very preterm born group compared to those born at term. It has been proposed that the recruitment of additional, less specialized brain regions may be an adaptive mechanisms employed by the brain to cope with highly demanding tasks (Just and Varma, 2007). In line with this suggestion, a functional MRI study reported increased activation in less task-specific brain regions in adults born very preterm compared to term-born peers when performing a response inhibition task (Lawrence et al., 2009). Similarly, the larger cluster of electrodes with strong positive associations between SWA and executive function abilities reported here for the very preterm compared to the term-born group may reflect such adaptive mechanism of the very preterm brain.

While it is well known that frontal brain regions are crucially involved in executive processes, the association between more SWA over central regions and poorer executive function abilities, which was particularly evident in the term-born group, is more difficult to interpret. Previously, SWA in this region has been associated with the development of simple and complex motor abilities in typically-developing children (Kurth et al., 2012). Interestingly, different studies have reported that reduced activity in certain brain regions while performing a task may be associated with good rather than poor performance. For example, single neuron recordings in the posterior cingulate in animals revealed that the decreased neuronal firing rate during a working memory task is associated with better performance (Hayden et al., 2009). In humans, various functional MRI studies have reported that the increased suppression of the default-mode network during 
task execution is associated with better performance (see Anticevic et al., 2012 for an overview). Accordingly, in adults born very preterm who performed comparably to term-born peers in a working-memory task, prominent suppression of the default-mode network during task execution was observed, particularly when task demands were high (Daamen et al., 2014). The association between less sleep SWA over central brain regions and better executive function abilities which was observed in the current study may reflect such processes and future studies using, for example, source localization should further elaborate on this issue.

In contrast to other neuroimaging methods, EEG assessments during sleep allow the investigation of brain activity independent of subject- and task-related factors inherent to wakefulness (Tononi and Cirelli, 2006). This may be of particular relevance when investigating neurodevelopment following very preterm birth: Significant group differences in, for example, executive function performance compared to term-born peers have been reported repeatedly (Aarnoudse et al., 2012; Burnett et al., 2015) and it has been suggested that performance differences between groups rather than birth status per se may alter brain activity assessed with functional MRI (Ment and Constable, 2007). The very preterm children and adolescents assessed in the current study experienced significant executive function deficits compared to their termborn peers despite normal intellectual and motor abilities. The assessment of the functional neuroanatomy of the impaired processes during sleep rather than waking allowed the unbiased identification of alterations and, consequently, may facilitate the understanding of neuronal mechanisms underlying these deficits.

No global alterations in the topographical distribution of sleep SWA were apparent in the very preterm individuals assessed in this study compared to their term-born peers, thus, suggesting similar general functional neuroanatomy in both groups. Importantly, using a highdensity EEG recording device allowed the investigation of localized patterns of brain activity in relation to cognitive abilities. This revealed alterations in the functional neuroanatomy of executive processes, confined to frontal and temporo-central brain areas, in individuals born very preterm. Hence, in the absence of major between-group differences in neuroanatomy, the investigation of the functional integrity of specific cognitive networks may be necessary to better understand potential underlying mechanisms of deficits. Similarly, in adults born very preterm, structural brain changes could not fully explain the altered activation patterns in response to executive-type tasks in a functional MRI study (Nosarti et al., 2009). Considering the 
aforementioned benefits of sleep EEG when investigating the functional neuroanatomy of cognitive processes, high-density recordings are particularly useful for evaluating local aspects of altered brain development following very preterm birth.

The slow waves dominating the surface EEG during deep sleep result from the synchronized oscillatory activity in the component neurons of the thalamocortical system (see Crunelli et al., 2015 for an overview). As the emergence of thalamocortical connections constitutes a key neurodevelopmental process during the last trimester of pregnancy (Volpe, 2009; Kostović and Judaš, 2010), very preterm birth, coinciding with this crucial period of brain development, puts the thalamocortical system at specific risk for adverse environmental impacts such as hypoxia/ischemia, inflammation and other neonatal complications (Volpe, 2009). Accordingly, impaired thalamic development and disrupted thalamocortical connectivity have been reported repeatedly in very preterm infants (Boardman et al., 2006; Counsell et al., 2007; Srinivasan et al., 2007; Smyser et al., 2010; Ball et al., 2012; Ball et al., 2013) and first findings suggest that such impairments of the thalamocortical system may underlie later neurodevelopmental deficits (Ball et al., 2015). In the current study, SWA topography in relation to executive function abilities was different between very preterm and term-born individuals. Hence, altered thalamocortical connectivity due to early disruptions of normal brain development may underlie these differences in the functional neuroanatomy of executive processes and potentially lead to the apparent executive function deficits. Similarly, sleep SWA has previously been shown to be closely associated with the integrity of white matter tracts of frontal brain regions and the corpus callosum in typical brain development (Buchmann et al., 2011a; Piantoni et al., 2013). White matter injuries are common after very preterm birth and may impede subsequent brain development (Volpe, 2003, 2009; de Kieviet et al., 2012). Importantly, diffuse white matter abnormalities have been associated with aberrant functional connectivity in executive networks in very preterm infants, thus, highlighting the close relationship between the structural and functional neuroanatomy of cognitive networks (He and Parikh, 2015). In the future, employing sleep SWA topography as a mapping tool of the functional neuroanatomy of cognitive networks in parallel to the assessment of their structural integrity may provide novel insight into how the brain copes with disruptions of typical developmental processes following very preterm birth.

Besides its potential to map the functional neuroanatomy of cognitive processes in typical and atypical brain development, sleep SWA also reflects neuroplastic processes within cortical 
networks: Sleep slow waves are tightly connected to the functional properties of synapses as they reflect the synchrony and synaptic strength of neuronal networks (Tononi and Cirelli, 2006). Importantly, SWA has been shown to increase as a function of the prior use of these networks during wakefulness, presumably reflecting neuroplastic processes (i.e., increased synaptic strength). For example, after typically-developing children and adolescents had learned a visuospatial task, SWA in the subsequent night was locally increased over parietal brain regions known to be involved in visuo-spatial learning (Wilhelm et al., 2014). Also, in typicallydeveloping adolescent boys, sleep SWA was increased after three weeks of intensive working memory training over brain regions which are known to be part of the core working memory network with the increase being related to improved performance after the training (Pugin et al., 2014). Sleep SWA, thus, seems to reflect both the short- and long-term use-dependent functional plasticity of cognitive networks. In the current study, a local increase of sleep SWA was observed in very preterm individuals compared to term-born peers with similar executive function abilities exclusively over frontal brain regions, which are known to be associated with executive processing. In line with the use-dependence of SWA, this local increase of SWA may reflect plastic mechanisms of the preterm brain to cope with the high demands placed on executive function abilities. In other words, to achieve similar performance as their term-born peers, very preterm individuals may strain executive function networks more strongly, which leads to increased synchrony and synaptic strength within the relevant networks and, subsequently, to the expression of more sleep SWA during the night. Results from several functional MRI studies may support this interpretation, as increased activation within relevant networks in response to different executive function tasks have been reported for children and adults born very preterm compared to term-born peers (Nosarti et al., 2006; Kalpakidou et al., 2014; Mürner-Lavanchy et al., 2014). In parallel, childhood and adolescence have been described as developmental periods in which the demands placed on executive function abilities by the environment increase markedly (Jacobson et al., 2011; Burnett et al., 2013). Those born very preterm may be particularly challenged (Luciana et al., 1999; Bayless and Stevenson, 2007; Wehrle et al., 2016) and the deficits in a variety of executive functions which become only evident under highly demanding conditions may be a quantitative marker of these load-dependent executive function deficits following very preterm birth (Wehrle et al., 2016). Compensational mechanisms of the very preterm brain, namely the stronger employment of networks, may help to cope with high 
demands and their failure may underlie the load-dependent deficits in executive processes exhibited by many adolescents born very preterm.

The findings of the current study suggest that not only the increased activation during taskexecution but also the sleeping brain may contribute to compensational processes in executive functioning in very preterm individuals. Many studies have previously provided evidence that sleep slow waves are crucial for functional recovery processes within sensory, motor and cognitive networks and that this may underlie the continuous capacity of the brain to adapt to the environment (Van Der Werf et al., 2009; Groeger et al., 2014; Tononi and Cirelli, 2014; Achermann and Borbély, 2015). Accordingly, high sleep SWA in those born very preterm may facilitate functional recovery of executive function networks and, thus, support optimal performance. Future studies will need to further investigate this issue (see also Limitations).

\subsection{Limitation}

The cross-sectional design of the current study prohibits the assessment of developmental aspects. As childhood and adolescence is a developmental period of major structural and functional reorganization of brain networks, longitudinal assessments including several assessments of cognitive abilities and brain activity are needed to investigate developmental trajectories and the occurrence of compensational mechanisms following very preterm birth. Such data may help to gain insight into how the preterm brain copes with early disruptions of normal development and to define time windows when children may be particularly susceptible to interventions. Also, other factors, for example sex, may affect the association between sleep SWA and executive processes and future studies should be designed to address this issue.

The participants of the current study only spent one night in the sleeping lab. This prohibited the systematic manipulation of executive function demands and the investigation of subsequent effects on sleep SWA. Instead, executive function performance under highly demanding conditions was used as a marker of load-dependent deficits and related to sleep SWA topography in a correlational manner. Future studies which record several nights of high-density EEG during sleep following performance assessments under varying executive function loads are needed to shed light on the causal role of high loads on executive function networks. 
Along the same line, executive functions were only assessed in the afternoon preceding sleep and no tasks were applied in the morning. Thus, this study cannot provide information on whether sleep and sleep SWA, indeed, actively contribute to functional recovery within executive function networks and, thus, support compensational processes.

The examiner who conducted the neurodevelopmental assessments was not blinded to the participants' birth status. While this could have induced an assessment bias, we are confident that the results are unaffected as the examiner is a trained neuropsychologist and all assessments were conducted in accordance with standardized manuals. Also, the examiner was unaware of the medical history of very preterm participants until neurodevelopmental assessments were completed to prevent potential effects on results. Still, future studies should aim at designing protocols for which blinding is feasible to fully exclude the possibility of any bias.

Our results provide evidence that executive function abilities are primarily related to sleep SWA as the association in frontal and centro-parietal brain regions was found to be frequencyspecific. As other characteristics of the sleep EEG, for example sleep spindles, have previously been shown to be related to cognitive abilities (see e.g., Fogel and Smith, 2011 for a review) and are known to reflect the integrity of the thalamocortical system as a whole (Steriade et al., 1993), future studies should investigate potential alterations following very preterm birth and whether this is related to cognitive deficits in this population.

\subsection{Conclusion}

This is the first study to show that sleep SWA assessed with high-density EEG is a valuable tool to map the altered functional neuroanatomy of executive processes following very preterm birth. Particularly, increased SWA was apparent in very preterm individuals compared to similarly performing term-born peers over frontal brain regions, pivotal parts of the executive function network. This may reflect the increased use of this network during the day and, thus, be a compensational mechanism of the preterm brain in response to high executive function demands. Load-dependent executive function deficits may be a consequence of inadequate functional plasticity when the demands exceed a certain level. The findings of this study add to the current knowledge on the functional neuroanatomy of executive processes following very 
preterm birth by investigating brain networks independent of confounding factors related to waking (e.g., attention allocation).

\section{Acknowledgements}

We would like to thank Alejandra Hüsser, Hadwig Speckbacher, Cléa Verrey, Hideo Breden, Laura Benz and Kirill Karlin for their support in data collection and data preprocessing, Liane Kaufmann for her advice in compiling the cognitive test battery and Angelina Maric and Carina Volk for their valuable input to the manuscript. A special thank goes to all the participating children, adolescents and their families.

This work was supported by the Zurich Center for Integrative Human Physiology (ZIHP), the Anna Mueller Grocholski Foundation, the Swiss National Science Foundation (PP00P3_135438), the Clinical Research Priority Program "Sleep and Health" of the University of Zurich and the "For Women in Science"-grant by L'Oréal Foundation, the Swiss Commission for UNESCO and the Swiss Academics for Arts and Sciences.

\section{References}

Aarnoudse CS, Duivenvoorden HJ, Weisglas N, Van Goudoever JB, Oosterlaan J. The profile of executive function in very preterm children at 4 to 12 years. Dev Med Child Neurol 2012; 54(3): 247-53.

Achermann P, Borbély A. Sleep homeostasis and models of sleep regulation. In: Kryger M, Roth T, Dement W, editors. Principles and practice of sleep medicine. 6th ed. Philadelphia: Elsevier; 2015. p. 377-87.

Anderson P. Assessment and development of executive function (EF) during childhood. Child Neuropsychol 2002; 8(2): 71-82.

Anticevic A, Cole MW, Murray JD, Corlett PR, Wang X-J, Krystal JH. The role of default network deactivation in cognition and disease. Trends in Cogn Sci 2012; 16(12): 584-92.

Aschenbrenner S, Tucha O, Lange KW. Regensburger Wortflüssigkeits-Test (RWT) [Regensburger verbal fluency test (RWT)]. Göttingen: Hogrefe Verlag; 2000. 
Ball G, Boardman JP, Aljabar P, Pandit A, Arichi T, Merchant N, et al. The influence of preterm birth on the developing thalamocortical connectome. Cortex 2013; 49(6): 1711-21.

Ball G, Boardman JP, Rueckert D, Aljabar P, Arichi T, Merchant N, et al. The effect of preterm birth on thalamic and cortical development. Cereb Cortex 2012; 22(5): 1016-24.

Ball G, Pazderova L, Chew A, Tusor N, Merchant N, Arichi T, et al. Thalamocortical Connectivity Predicts Cognition in Children Born Preterm. Cereb Cortex 2015: bhu331.

Bayless S, Stevenson J. Executive functions in school-age children born very prematurely. Early Hum Dev 2007; 83(4): 247-54.

Boardman JP, Counsell SJ, Rueckert D, Kapellou O, Bhatia KK, Aljabar P, et al. Abnormal deep grey matter development following preterm birth detected using deformation-based morphometry. Neuroimage 2006; 32(1): 70-8.

Buchmann A, Kurth S, Ringli M, Geiger A, Jenni OG, Huber R. Anatomical markers of sleep slow wave activity derived from structural magnetic resonance images. J Sleep Res 2011a; 20(4): 506-13.

Buchmann A, Ringli M, Kurth S, Schaerer M, Geiger A, Jenni OG, et al. EEG sleep slow-wave activity as a mirror of cortical maturation. Cerebral Cortex 2011b; 21(3): 607-15.

Burnett AC, Scratch SE, Lee KJ, Cheong J, Searle K, Hutchinson E, et al. Executive Function in Adolescents Born< 1000 g or $<28$ Weeks: A Prospective Cohort Study. Pediatrics 2015; 135(4): e826-e34.

Cambridge neuropsychological test automated battery. Cambridge (UK): Cambridge Cognition Ltd; 2004.

Counsell SJ, Dyet LE, Larkman DJ, Nunes RG, Boardman JP, Allsop JM, et al. Thalamo-cortical connectivity in children born preterm mapped using probabilistic magnetic resonance tractography. Neuroimage 2007; 34(3): 896-904.

Crunelli V, David F, Lörincz ML, Hughes SW. The thalamocortical network as a single slow wave-generating unit. Curr Opin Neurobiol 2015; 31: 72-80.

Curtis WJ, Zhuang J, Townsend EL, Hu X, Nelson CA. Memory in early adolescents born prematurely: a functional magnetic resonance imaging investigation. Dev Neuropsychol 2006; 29(2): 341-77.

Daamen M, Bäuml JG, Scheef L, Sorg C, Busch B, Baumann N, et al. Working memory in preterm-born adults: Load-dependent compensatory activity of the posterior default mode network. Hum Brain Mapp 2014. 
De Gennaro L, Ferrara M, Vecchio F, Curcio G, Bertini M. An electroencephalographic fingerprint of human sleep. Neuroimage 2005; 26(1): 114-22.

De Gennaro L, Marzano C, Fratello F, Moroni F, Pellicciari MC, Ferlazzo F, et al. The electroencephalographic fingerprint of sleep is genetically determined: a twin study. Ann Neurol 2008; 64(4): 455-60.

de Kieviet JF, Zoetebier L, Van Elburg RM, Vermeulen RJ, Oosterlaan J. Brain development of very preterm and very low-birthweight children in childhood and adolescence: a meta-analysis. Dev Med Child Neurol 2012; 54(4): 313-23.

Diamond A. Normal development of prefrontal cortex from birth to young adulthood: Cognitive functions, anatomy, and biochemistry. In: Stuss D, Knight R, editors. Principles of frontal lobe function. New York: Oxford University Press; 2002. p. 466-503.

Finelli LA, Achermann P, Borbély AA. Individual 'fingerprints' in human sleep EEG topography. Neuropsychopharmacology 2001; 25: S57-S62.

Fogel SM, Smith CT. The function of the sleep spindle: a physiological index of intelligence and a mechanism for sleep-dependent memory consolidation. Neurosci Biobehav Rev 2011; 35(5): 1154-65.

Geiger A, Huber R, Kurth S, Ringli M, Achermann P, Jenni OG. Sleep electroencephalography topography and children's intellectual ability. Neuroreport 2012; 23(2): 93-7.

Griffiths ST, Gundersen H, Neto E, Elgen I, Markestad T, Aukland SM, et al. fMRI: blood oxygen level-dependent activation during a working memory-selective attention task in children born extremely preterm. Pediatr Res 2013; 74(2): 196-205.

Groeger JA, Stanley N, Deacon S, Dijk D-J. Dissociating effects of global SWS disruption and healthy aging on waking performance and daytime sleepiness. Sleep 2014; 37(6): 112742.

Hayden BY, Smith DV, Platt ML. Electrophysiological correlates of default-mode processing in macaque posterior cingulate cortex. PNAS 2009; 106(14): 5948-53.

He L, Parikh NA. Aberrant Executive and Frontoparietal Functional Connectivity in Very Preterm Infants with Diffuse White Matter Abnormalities. Pediatr Neurol 2015.

Huber R, Ghilardi MF, Massimini M, Tononi G. Local sleep and learning. Nature 2004; 430(6995): 78-81.

Iber C, Ancoli-Israel S, Chesson A, Quan S. The AASM Manual for the Scoring of Sleep and Associated Events. Westchester, IL: American Academy of Sleep Medicine; 2007. 
Jacobson LA, Williford AP, Pianta RC. The role of executive function in children's competent adjustment to middle school. Child Neuropsychol 2011; 17(3): 255-80.

Just MA, Varma S. The organization of thinking: What functional brain imaging reveals about the neuroarchitecture of complex cognition. Cogn Affect Behav Neurosci 2007; 7(3): 15391.

Kalpakidou AK, Allin MP, Walshe M, Giampietro V, McGuire PK, Rifkin L, et al. Functional Neuroanatomy of Executive Function after Neonatal Brain Injury in Adults Who Were Born Very Preterm. PloS one 2014; 9(12): e113975.

Kostović I, Judaš M. The development of the subplate and thalamocortical connections in the human foetal brain. Acta Paediatrica 2010; 99(8): 1119-27.

Kurth S, Ringli M, Geiger A, LeBourgeois M, Jenni OG, Huber R. Mapping of cortical activity in the first two decades of life: a high-density sleep electroencephalogram study. J Neurosci 2010; 30(40): 13211-9.

Kurth S, Ringli M, LeBourgeois MK, Geiger A, Buchmann A, Jenni OG, et al. Mapping the electrophysiological marker of sleep depth reveals skill maturation in children and adolescents. Neuroimage 2012; 63(2): 959-65.

Largo RH, Fischer JE, Caflisch JA, Jenni OG. Zurich Neuromotor Assessment. Zurich: AWE Verlag; 2007.

Largo RH, Pfister D, Molinari L, Kundu S, Lipp A, Duc G. Significance of prenatal, perinatal and postnatal factors in the development of AGA preterm infants at five to seven years. Dev Med Child Neurol 1989; 31(4): 440-56.

Latal B. Prediction of neurodevelopmental outcome after preterm birth. Pediatr Neurol 2009; 40(6): 413-9.

Lawrence EJ, Rubia K, Murray RM, McGuire PK, Walshe M, Allin M, et al. The neural basis of response inhibition and attention allocation as mediated by gestational age. Hum Brain Mapp 2009; 30(3): 1038-50.

Luciana M, Lindeke L, Georgieff M, Mills M, Nelson CA. Neurobehavioral evidence for working-memory deficits in school-aged children with histories of prematurity. Dev Med Child Neurol 1999; 41(8): 521-33.

Lustenberger C, Huber R. High density electroencephalography in sleep research: potential, problems, future perspective. Front Neurol 2012; 3. 
Lustenberger C, Mouthon A, Tesler N, Kurth S, Ringli M, Buchmann A, et al. Developmental trajectories of EEG sleep slow wave activity as a marker for brain and motor skill development during adolescence. Dev Psychobiol 2016; 9999.

Lustenberger C, Wehrle F, Tüshaus L, Achermann P, Huber R. The Multidimensional Aspects of Sleep Spindles and Their Relationship to Word-Pair Memory Consolidation. Sleep 2014; 38(7): 1093-103.

Ment LR, Constable RT. Injury and recovery in the developing brain: evidence from functional MRI studies of prematurely born children. Nat Clin Pract Neurol 2007; 3(10): 558-71.

Ment LR, Hirtz D, Hüppi PS. Imaging biomarkers of outcome in the developing preterm brain. Lancet Neurol 2009; 8(11): 1042-55.

Miyake A, Friedman NP, Emerson MJ, Witzki AH, Howerter A, Wager TD. The unity and diversity of executive functions and their contributions to complex "frontal lobe" tasks: A latent variable analysis. Cognitive Psychol 2000; 41(1): 49-100.

Murphy K, Garavan H. Artifactual fMRI group and condition differences driven by performance confounds. Neuroimage 2004; 21(1): 219-28.

Mürner-Lavanchy I, Ritter B, Spencer-Smith M, Perrig W, Schroth G, Steinlin M, et al. Visuospatial working memory in very preterm and term born children-Impact of age and performance. Dev Cognitive Neurosci 2014; 9: 106-16.

Nichols TE, Holmes AP. Nonparametric permutation tests for functional neuroimaging: a primer with examples. Hum Brain Mapp 2002; 15(1): 1-25.

Nosarti C, Giouroukou E, Healy E, Rifkin L, Walshe M, Reichenberg A, et al. Grey and white matter distribution in very preterm adolescents mediates neurodevelopmental outcome. Brain 2008; 131(1): 205-17.

Nosarti C, Rubia K, Smith AB, Frearson S, Williams SC, Rifkin L, et al. Altered functional neuroanatomy of response inhibition in adolescent males who were born very preterm. Dev Med Child Neurol 2006; 48(04): 265-71.

Nosarti C, Shergill SS, Allin MP, Walshe M, Rifkin L, Murray RM, et al. Neural substrates of letter fluency processing in young adults who were born very preterm: alterations in frontal and striatal regions. Neuroimage 2009; 47(4): 1904-13.

Østgård HF, Sølsnes AE, Bjuland KJ, Rimol LM, Martinussen M, Brubakk A-M, et al. Executive function relates to surface area of frontal and temporal cortex in very-low-birth-weight late teenagers. Early human development 2016; 95: 47-53. 
Petermann F, Petermann U. Hamburg-Wechsler Intelligenztest für Kinder-IV (HAWIK-IV) [Hamburg-Wechsler-Intelligence Test for children (HAWIK-IV)]. Bern: Huber; 2006.

Piantoni G, Poil S-S, Linkenkaer-Hansen K, Verweij IM, Ramautar JR, Van Someren EJ, et al. Individual differences in white matter diffusion affect sleep oscillations. J Neurosci 2013; 33(1): 227-33.

Pugin F, Metz A, Wolf M, Achermann P, Jenni O, Huber R. Local Increase of Sleep Slow Wave Activity After Three Weeks of Working Memory Training in Children and Adolescents. Sleep 2014.

Ringli M, Souissi S, Kurth S, Brandeis D, Jenni OG, Huber R. Topography of sleep slow wave activity in children with attention-deficit/hyperactivity disorder. Cortex 2013; 49(1): 3407.

Skranes J, Lohaugen GC, Martinussen M, Indredavik MS, Dale AM, Haraldseth O, et al. White matter abnormalities and executive function in children with very low birth weight. Neuroreport 2009; 20(3): 263-6.

Skranes J, Løhaugen GC, Evensen KAI, Indredavik MS, Haraldseth O, Dale AM, et al. Entorhinal cortical thinning affects perceptual and cognitive functions in adolescents born preterm with very low birth weight (VLBW). Early Hum Dev 2012; 88(2): 103-9.

Smyser CD, Inder TE, Shimony JS, Hill JE, Degnan AJ, Snyder AZ, et al. Longitudinal analysis of neural network development in preterm infants. Cereb Cortex 2010: bhq035.

Srinivasan L, Dutta R, Counsell SJ, Allsop JM, Boardman JP, Rutherford MA, et al. Quantification of deep gray matter in preterm infants at term-equivalent age using manual volumetry of 3-tesla magnetic resonance images. Pediatrics 2007; 119(4): 759-65.

Steriade M, McCormick DA, Sejnowski TJ. Thalamocortical oscillations in the sleeping and aroused brain. Science 1993; 262(5134): 679-85.

Taylor HG, Filipek PA, Juranek J, Bangert B, Minich N, Hack M. Brain volumes in adolescents with very low birth weight: effects on brain structure and associations with neuropsychological outcomes. Dev Neuropsychol 2011; 36(1): 96-117.

Tononi G, Cirelli C. Sleep function and synaptic homeostasis. Sleep Med Rev 2006; 10(1): 4962.

Tononi G, Cirelli C. Sleep and the price of plasticity: from synaptic and cellular homeostasis to memory consolidation and integration. Neuron 2014; 81(1): 12-34. 
Uddin LQ, Supekar K, Menon V. Typical and atypical development of functional human brain networks: insights from resting-state FMRI. Front Syst Neurosci 2010; 4: 21.

Ullman H, Almeida R, Klingberg T. Structural maturation and brain activity predict future working memory capacity during childhood development. J Neurosci 2014; 34(5): 15928.

Van Der Werf YD, Altena E, Schoonheim MM, Sanz-Arigita EJ, Vis JC, De Rijke W, et al. Sleep benefits subsequent hippocampal functioning. Nat Neurosci 2009; 12(2): 122.

Volpe JJ. Cerebral white matter injury of the premature Infant - more common than you think. Pediatrics 2003; 112(1): 176-80.

Volpe JJ. The encephalopathy of prematurity - brain injury and impaired brain development inextricably intertwined. Semin Pediatr Neurol; 2009: Elsevier; 2009. p. 167-78.

Wehrle FM, Kaufmann L, Benz LD, Huber R, O'Gorman RL, Latal B, et al. Very preterm adolescents show impaired performance with increasing demands in executive function tasks. Early Hum Dev 2016; 92: 37-43.

Wilhelm I, Kurth S, Ringli M, Mouthon A-L, Buchmann A, Geiger A, et al. Sleep slow-wave activity reveals developmental changes in experience-dependent plasticity. J Neurosci 2014; 34(37): 12568-75. 